\title{
A MULTI-SPACECRAFT VIEW OF SOLAR-ENERGETIC-PARTICLE ONSETS IN THE 1977 NOVEMBER 22 EVENT
}

\author{
Donald V. Reames \\ Institute for Physical Science and Technology, \\ University of Maryland, College Park, MD 20742 \\ dvreames@umd.edu \\ and \\ Nand Lal \\ NASA Goddard Space Flight Center \\ Greenbelt, MD 20771
}

Revision of ApJ80670, resubmitted to Astrophysical Journal 


\begin{abstract}
We examine the onset timing of solar energetic particles (SEPs) in the large ground-level event (GLE) of 1977 November 22 as observed from six spacecraft at four distinct solar longitudes. In most cases, it was possible to use velocity dispersion of the energetic protons to fix the solar particle release (SPR) time and the path-length traveled by the initial particle burst from each solar longitude. We find that the SPR times do depend upon solar longitude, being earliest for spacecraft that are magnetically wellconnected to the source region, and later for longitudes on the flanks of the outward driven shock wave. The earliest SPR time occurs well after peak photon emission from the associated Ha flare. These observations are consistent with conclusions derived from single-longitude observations of different GLE events.
\end{abstract}

Subject headings: acceleration of particles -shock waves-Sun: coronal mass ejections (CMEs) - Sun: particle emission - Sun: radio radiation 


\section{INTRODUCTION}

There are large solar energetic particle (SEP) events with extremely hard energy spectra having $\mathrm{GeV}$ protons that produce radiation in the Earth's atmosphere that can be detected when it penetrates to ground level. Seventy of these ground-level events (GLEs) have been identified since 1942 (Cliver et al. 1982; Cliver 2006). In their classic review, Cliver et al. (1982) found that the earliest relativistic protons could be accelerated more than $20 \mathrm{~min}$ after the end of the flash phase of the associated flare. They concluded that these observations suggested acceleration at a shock front as it crosses onto open magnetic field lines connecting to Earth. The idea of particle acceleration at the shock wave driven out from the Sun by coronal mass ejections (CMEs) is now commonly invoked to explain many features of large SEP events (see reviews Reames 1990, 1995, 1999, 2002, 2009ab; Kahler 1992, 1994, 2001; Gosling 1993; Lee 1997, 2005; Tylka 2001; Tylka et al. 2005; Tylka \& Lee 2006).

The inferred initial departure time of SEPs from the Sun depends directly on their path length along the magnetic field line to the observing spacecraft. The nominal path length of $\sim 1.2 \mathrm{AU}$ along the Parker spiral to Earth is often exceeded when CMEs disrupt this nominal pattern. At times, complicated field geometry can be mapped by tracking the frequency and direction of kilometric type III radio emission by electrons streaming outward from the Sun (see e.g. Reames \& Stone 1986). Studies of onset timing of impulsive electron events inside magnetic clouds have shown magnetic path lengths varying from $\sim 1.2 \mathrm{AU}$ near the center of the cloud to $\sim 3 \mathrm{AU}$ at its periphery (Larson et al 1997).

Fortunately, onset times for particles of different energy provide a direct measurement of the magnetic path length since the particles arrive inversely ordered by their 
velocity, i.e. the fastest particles arrive first, etc. Thus the partcle transit time equals the path length divided by the particle velocity, $v$, for unscattered particles propagating along the field, and the fitted line on a plot of onset time $v s . v^{-1}$ has the path length as its slope and the earliest solar particle release (SPR) time at the Sun as its intercept. Following an early study of both impulsive SEP events and GLEs by Tylka et al. (2003), recent papers (Reames 2009a and b, hereinafter papers I and II) have described this technique in detail, discussed its limitations, and determined the initial SPR times and path lengths for 30 of the 45 GLEs that have occurred since 1973 using measurements from spacecraft near Earth.

Plotting the height of the CME-driven shock at SPR time as a function of the relative solar longitude of the observation, the ensemble of GLEs showed a pattern of heights that were as low as $\sim 2 \mathrm{R}_{\mathrm{S}}$ for well-connected events but increasing in height on the flanks of the CME (paper II, see also Cliver, Kahler, \& Reames 2004). Since each GLE was observed from only one solar longitude, it was not possible to verify that this pattern was appropriate for any individual event.

It is important to realize that the path length is an experimentally determinable quantity and not an adjustable parameter that can be increased arbitrarily to match particle onsets with interesting photon peak times. Conversely, it is also important to note that the assumption of a constant path length, like 1.2 AU, will make some events appear to originate much later and farther out from the Sun than they actually do.

In papers I and II different GLEs were observed from Earth, a single solar longitude. In this paper we take the opposite perspective by studying the onset timing of a single GLE event, of 1977 November 22, viewed from several spacecraft, IMP-7 and -8, 
Voyager-1, and -2, and Helios-1, and -2, representing four distinct solar longitudes and different radial distances from the Sun. While some of the measurements are more limited in scope than we would like, the observation of a GLE at relatively high time resolution, over such a convenient spatial pattern is uncommon, and we have taken the opportunity to measure and report it. We were especially motivated to resurrect historic data from this event by the fact that no comparable present or future study of GLEs is possible with more-modern spacecraft that are presently active; either they are too widely separated or are too closely grouped near Earth.

\section{ANALYSIS OF SEP ONSET TIMES}

Figure 1 shows the spatial distribution of the spacecraft in the ecliptic plane and the magnetic field lines connecting them to the Sun, as deduced from the solar wind speed observed at each spacecraft. The CME source, assumed to coincide with the Ha flare location at N24W40 as viewed from Earth (IMP-7, -8), is directed downward in the figure and an illustrative shock wave is shown.

Figure 2 shows proton intensities observed on 1977 November 22 by $I M P-8$ and by the two Voyager spacecraft. Since the Voyager spacecraft do not spin, we have summed data from the 2 double-ended High Energy Telescopes (HETs) and/or the 4 Low Energy Telescopes (LETs) that have widely separated look directions (Stone et al. 1977; Stilwell et al. 1979), to insure that we include directions that are aligned with the magnetic field as well as possible. Since the two Voyager spacecraft are relatively close together, for our purposes (see Table 1 below), we have also summed intensities at corresponding energies from both spacecraft in the figure. In contrast, the two spinning IMP spacecraft have been treated as providing independent measures of onset times even though they are 
both near Earth. This retains the treatment used in paper II, where data from IMP-7 and 8 are combined with neutron-monitor data to give the best near-Earth fit to the velocity dispersion.

Figure 3 shows relative intensities of protons measured by Helios-1 and -2 (Stilwell et al. 1975; Trainor et al. 1975) in the 1977 November 22 event. For Helios-1 we have information that is more limited since pulse-height data, required to specify the energy of each proton unambiguously, are not available. Rates that respond primarily to lowerenergy protons are partially contaminated by high-energy protons, so only the highestenergy proton onset times are valid and are shown in the figure. For Helios-2, there are sufficient pulse-height data to calculate intensities for two lower-energy intervals, as shown.

Figure 4 shows the onset timing data for Voyager, IMP-7, IMP-8, and Helios-2 along with the fitted lines for each spacecraft group. The fit line for IMP is that originally obtained in paper II. The SPR times at the Sun and path lengths for each case are shown in Table 1. The table also lists the radius, $R_{\mathrm{SC}}$, latitude, $\theta_{\mathrm{HGI}}$, and longitude, $\boldsymbol{\Psi}_{\mathrm{HGI}}$, of each spacecraft in heliographic inertial (HGI) coordinates, and the solar wind speed, $V_{\mathrm{SW}}$, at each spacecraft near the time of SEP onset, as well as the shock transit speed, $V_{\text {Shock} \text {. }}$

The SEP path lengths shown in Table 1 are reasonably close to the lengths along the Parker spiral for this event. At Helios, nearer the Sun, it is not surprising that the path lengths are close to the radial and Parker spiral distances. However, at Earth the path length of $1.3 \pm 0.1 \mathrm{AU}$ is slightly larger than the Parker spiral length of $1.2 \mathrm{AU}$, and at Voyager, at $1.5 \mathrm{AU}$, the path length of $1.9 \pm 0.1 \mathrm{AU}$ is also close to the Parker spiral 
length of $2.0 \mathrm{AU}$. This circumstance is relatively rare (see paper II) and probably results from the quiet conditions that exist for many days prior to the event.

\section{SPATIAL DISTRIBUTION OF THE EVENT SOURCES}

To estimate the location of the CME-driven shock at initial SPR time for each spacecraft, we follow the procedure that was used and verified in paper I. This assumes that a typical metric type II onset frequency of $\sim 100 \mathrm{MHz}$ corresponds to a radius of $\sim 1.5$ $\mathrm{R}_{\mathrm{S}}$ (e.g. Kundu 1965) where shock formation began. Subsequently, the CME or shock speed multiplied by the delay of the SPR time after type II onset, $D t$, then gave the height of the shock corresponding to the initial SPR time, thus $R(\mathrm{SPR})=1.5+V_{\text {Shock. }} \mathrm{X} D t . \quad D t$, and $R(\mathrm{SPR})$ are included in Table 1 . These values of $R(\mathrm{SPR})$, determined similarly, compared favorably with heights determined from observed CME height-time plots as discussed in paper I. This procedure allows determination of the SPR height when CME observations are not available, as was the case in 1977.

For the 1977 November 22 event, we assume a source location at the Ha flare site N24W40 on the Sun and a shock speed of $588 \mathrm{~km} / \mathrm{s}$ (see Discussion below) that was determined by its transit time to Helios-2. As in earlier references, we assume the shock speed to be independent of longitude. According to Solar Geophysical Data, radio type V emission began at 0959 UT on November 22 (0950.7 UT at the Sun). An onset time of type II emission was not officially reported for this event, but observers at Weissenau Observatory reported it to be masked by the intense type IV emission at 1001.7 UT (0953.4 UT at Sun) that "overlaps type II" (Urbarz 1990). We use this time as a reference time for the onset of the type II burst. 
Table I and Figure 5 show the radial height of the shock at SPR time, $R(\mathrm{SPR})$, vs. $\Phi$, the solar source longitude relative to the footpoint of the field line from the observation. Note that the angle $\Phi$, as defined in paper II, increases toward the East. In paper II a first-order fit of $R(\mathrm{SPR}) v s . \Phi^{2}$ was used as an attempt to characterize the overall behavior of the data from the ensemble of GLE events, emphasizing the largest ones. For comparison, this fit, $R(\mathrm{SPR})=2.08+\left(\Phi / 36.4^{0}\right)^{2}$, appears as the parabola in Figure 5 .

\section{DISCUSSION}

We have found that the shape of $R(\mathrm{SPR})$ vs. $\Phi$ in a single GLE event is generally similar to that found for the ensemble of events in paper II. While the precise shape cannot be measured with observations at only 4 longitudes, the general rise if $R(\mathrm{SPR})$ on the flanks of the CME, seen in paper II, is confirmed. This consistency, using measurements at $\sim 0.6,1.0$, and $\sim 1.5 \mathrm{AU}$, suggests that radial position of the spacecraft, and the associated variation in the magnetic path length, is not a significant factor.

In all of the foregoing we have assumed that the shock speed is independent of solar longitude. The shock arrived at $I M P-8$ on November 25 at 1212:46 UT giving a transit speed of $560 \mathrm{~km} \mathrm{~s}^{-1}$, and at Voyager-1, on nearly the same longitude as Earth, at 2225 UT on November 27 with a transit speed of $474 \mathrm{~km} \mathrm{~s}^{-1}$. The shock arrived at $\mathrm{He}$ lios-2 on November 24 at 0611 UT indicating a transit speed of $588 \mathrm{~km} \mathrm{~s}^{-1}$ and was seen at Helios-1 on November 24 at 2227 UT indicating $468 \mathrm{~km} \mathrm{~s}^{-1}$ (Volkmer \& Neubauer 1985). This indicates some slowing of the shock, especially beyond $1 \mathrm{AU}$, as well as possible longitude dependence with a speed decrease on the flanks seen by Helios-1 (note that Helios- 1 is $79^{\circ}$ East of the source longitude). Of course, ideally we would like to 
know the shock speed at the footpoint longitude of each field line, not the longitude of the spacecraft itself, which is far to the East.

Sheeley et al. (1985) compared coronagraph observations of CME speed with in si$t u$ measurements on Helios. From their data we find a mean value of $V_{\mathrm{CME}} / V_{\text {transit }}=$ $1.01 \pm 0.07$, but the standard deviation of a single measurement is $33 \%$. Thus, using the shock speed at Helios-2 as our source shock speed seems reasonable. A higher shock speed would cause all the points in Figure 5 to move to a higher altitude, although the general shape of the distribution would remain the same. In fact, even doubling the shock speed would only add $\sim 1 \mathrm{R}_{\mathrm{S}}$ to the values of $R(\mathrm{SPR})$ for the magnetically wellconnected spacecraft. However, a decrease of shock speed on the flanks would flatten the pattern and might even explain the rise in $R(\mathrm{SPR})$ on the flanks entirely. It is likely that shock speeds do vary with longitude, latitude, and radius, but that information is not available for our event, and a general study of such variations with multi-spacecraft observations is unavailable and is beyond the scope of this paper.

The analysis technique in this and earlier papers assumes a single onset time for the shock as well as a single shock speed, while, in fact, both may depend on longitude as does the SPR time. Measurements of the shocks (or CMEs) are inadequate to fully explore the longitude dependence we find in the SPR time.

\section{CONCLUSIONS}

We have examined the initial release times of energetic solar particles observed by six spacecraft on four distinct longitudes in the 1977 November 22 SEP event. In most of the observations, SPR times and magnetic path lengths from the Sun to Earth are well determined, independently of connection longitude. Significantly, the time of the initial 
particle injection is different at different solar longitudes. In addition, the SPR times at all longitudes are more than $21 \mathrm{~min}$ after the earliest radio emission, $35 \mathrm{~min}$ after the $\mathrm{Ha}$ onset, and 13 min after Ha emission has peaked and begun its decline.

When we interpret the SPR time in terms of a height of initial acceleration in the corona, the pattern of height $v s$. longitude is generally consistent with shock acceleration over a broad spatial region with heights rising, and/or shock speeds falling, for longitudes on the flanks of the shock. This pattern is already seen in the SPR times themselves, which are not subject to uncertainties in the shock speed. This is the same pattern as was previously found in the ensemble of near-Earth measurements of many GLE events in paper II. We point out that a variation of shock speed with longitude would contribute to this pattern.

We thank Bryant Heikkila for his assistance in obtaining data from IMP and Voyager spacecraft. We thank Daniel Berdichevsky for a helpful discussion of Helios shock data and are especially grateful to Ed Cliver for helpful suggestions regarding the radio observations. We also thank Frank McDonald, Chee Ng, and Allan Tylka, for helpful discussions and for their comments on this paper.

This work was funded in part by NASA grant NNX08AQ02G. 
Cliver, E. W. 2006, ApJ 639, 1206

\section{REFERENCES}

Cliver, E. W., Kahler, S. W., \& Reames, D. V., 2004, ApJ 605, 902

Cliver, E. W., Kahler, S. W., Shea, M. A., \& Smart, D. F. 1982, ApJ 260, 362

Gosling, J. T. 1993, J. Geophys. Res., 98, 18937

Kahler, S. W. 1992, Ann. Rev. Astron. Astrophys. 30, 113

Kahler, S. W. 1994, ApJ, 428, 837

Kahler, S. W. 2001, J. Geophys. Res. 106, 20947

Kundu, M. R. 1965, Solar Radio Astronomy (New York: John Wiley and Sons).

Larson, D. E., et al. 1997, GRL 24, 1911

Lee, M. A. 1997, in: Coronal Mass Ejections, edited by N. Crooker, J. A. Jocelyn, J. Feynman, Geophys. Monograph 99 (AGU press), 227

Lee, M. A. 2005, ApJS, 158, 38

Reames, D. V., 1990, ApJS 73, 235

Reames, D. V. 1995, Revs. Geophys. (Suppl.) 33, 585

Reames, D. V. 1999, Space Sci. Revs., 90, 413

Reames, D. V. 2002, ApJ 571, L63

Reames, D. V. 2009a, ApJ 693, 812 (paper I)

Reames, D. V. 2009b, ApJ 706, 844 (paper II)

Reames, D. V., \& Stone, R. G., 1986, ApJ 308, 902

Sheeley, N. R., Jr., Howard, R. A., Koomen, M. J., Michels, D. J., Schwenn, R., Mühlhäuser, K. H., \& Rosenbauer, H., 1985, J. Geophys. Res,. 90, 163.

Stilwell, D. E., et. al. 1979, IEEE Trans. Nucl. Sci. NS-26, 513 
Stilwell, D. E.; Joyce, R. M.; Teegarden, B. J.; Trainor, J. H.; Streeter, G.; Bernstein, J. 1975, IEEE Trans. Nucl. Sci. NS-22, 570

Stone, E. C., Vogt, R. E., McDonald, F. B., Teegarden, B. J., Trainor, J. H., Jokipii, J. R., \& Webber, W. R. 1977, Space Sci. Revs., 21, 355.

Trainor, J. H., Stilwell, D. E., Joyce, R. M., Teegarden, B. J., \& White, H. O., Jr., 1975, Raumfahrtforschung 19, 258

Tylka, A. J. 2001, J. Geophys Res. 106, 25333

Tylka, A. J., Cohen, C.M.S., Dietrich, W. F., Krucker,, S., McGuire, R. E., Mewaldt, R. A. Ng, C. K., Reames, D. V., and Share, G. H., 2003, , Proc. $28^{\text {th }}$ Int. Cosmic Ray Conf., 3305

Tylka, A. J., Cohen, C. M. S., Dietrich, W. F., Lee, M. A., Maclennan, C. G., Mewaldt, R. A., Ng, C. K., and Reames, D. V. 2005, ApJ 625, 474

Tylka, A. J., \& Lee, M. A. 2006, ApJ 646, 1319

Urbarz, H.W. 1990, “Type II Solar Radio Bursts Recorded at Weissenau 1966-1987” Report UAG-98, (Boulder, CO: World Data Center A)

Volkmer, P. M., Neubauer, F. M. 1985, Annales Geophysicae 3, 1 
Table 1 Solar Particle Release and Related Properties seen from Different Spacecraft

\begin{tabular}{|c|c|c|c|c|c|c|c|c|c|c|}
\hline Spacecraft & $\begin{array}{l}R_{\mathrm{SC}} \\
(\mathrm{AU})\end{array}$ & $\begin{array}{c}\Theta_{H G I} \\
(\text { deg })\end{array}$ & $\begin{array}{c}\Psi_{\mathrm{HGI}} \\
(\mathrm{deg})\end{array}$ & $\begin{array}{c}V_{\mathrm{SW}} \\
(\mathrm{km} / \mathrm{s})\end{array}$ & $\begin{array}{c}V_{\text {shock }} \\
(\mathrm{km} / \mathrm{s})\end{array}$ & $\begin{array}{c}\text { Initial } \\
\text { SPR time } \\
\text { at Sun(UT) }\end{array}$ & $\begin{array}{l}\text { SEP Path } \\
\text { Length } \\
(\mathrm{AU})\end{array}$ & $\begin{array}{c}D t \\
(\min )\end{array}$ & $\begin{array}{c}\Phi \\
(\operatorname{deg})\end{array}$ & $\begin{array}{c}R(\mathrm{SPR}) \\
\left(\mathrm{R}_{\mathrm{S}}\right)\end{array}$ \\
\hline Helios 1 & 0.658 & 6.0 & 305.0 & 340.9 & 468 & $1024 \pm 5$ & $0.66 \pm 0.10$ & $30.6 \pm 5$ & $34.8 \pm 0.7$ & $3.04 \pm 0.24$ \\
\hline Helios 2 & 0.598 & 2.9 & 336.2 & 285.5 & 588 & $1011.9 \pm 1.3$ & $0.60 \pm 0.03$ & $18.5 \pm 1.3$ & $-0.2 \pm 1.7$ & $2.43 \pm 0.07$ \\
\hline IMP 7 & 1.00 & 2.0 & 344.2 & 331 & 560 & $1014.5+3.5$ & $1.31+0.09$ & $21.1+3.5$ & $-29.5+2.1$ & $2.56+0.17$ \\
\hline IMP 8 & 1.00 & 2.0 & 344.2 & 331 & 560 & $1014.5 \pm 3.5$ & $1.31 \pm 0.09$ & & $-29.3 \pm 2.1$ & $\angle .36 \pm 0.17$ \\
\hline Voyager 1 & 1.51 & 3.2 & 342.9 & 364.0 & 474 & $1044.0 \pm 6.7$ & $1.88 \pm 0.12$ & $50.6 \pm 6.7$ & $-55.1 \pm 1.3$ & $4.05 \pm 0.32$ \\
\hline Voyager 2 & 1.53 & 2.9 & 343.8 & 365.1 & 474 & & & & & \\
\hline
\end{tabular}




\section{FIGURE CAPTIONS}

Figure 1. The configuration of spacecraft and their magnetic connections to the Sun are shown in the ecliptic plane. A shock wave at the source longitude is shown projected downward in the figure.

Figure 2. The upper panel shows relative intensities of protons of various energy intervals vs. time as observed by IMP 8 at the 1977 November 22 GLE event onset. The lower panel shows similar data summed for Voyager 1 and 2. The solar location of the event, seen from Earth, is flagged at the time of Ha maximum.

Figure 3. The lower panel shows relative intensities of protons of the available energies vs. time as observed by Helios 2 at the 1977 November 22 GLE event onset. The upper panel shows high-energy proton intensities observed by Helios 1. The solar location of the event, seen from Earth, is flagged at the time of the Ha maximum.

Figure 4. A velocity dispersion plot shows onset times for protons of various energies vs. $v^{-1}$ at the various spacecraft indicated, as well as fitted lines for the data of each spacecraft location (see text). Radio onset time and the time of soft Ha onset are shown.

Figure 5. The delay of the SPR time after type II onset, $D t$, (upper panel) and the radial height of the shock at SPR time, $R(\mathrm{SPR})$, (lower panel) are shown as a function $\Phi$, the solar source longitude relative to the footpoint of the field line from the spacecraft. Please note that $\Phi$ increases from West to East, as was defined in paper II. The parabola is a first order fit of $R(\mathrm{SPR})$ vs $\Phi^{2}$, namely $R=2.08+\left(\Phi / 36.4^{0}\right)^{2}$, obtained in paper II. 


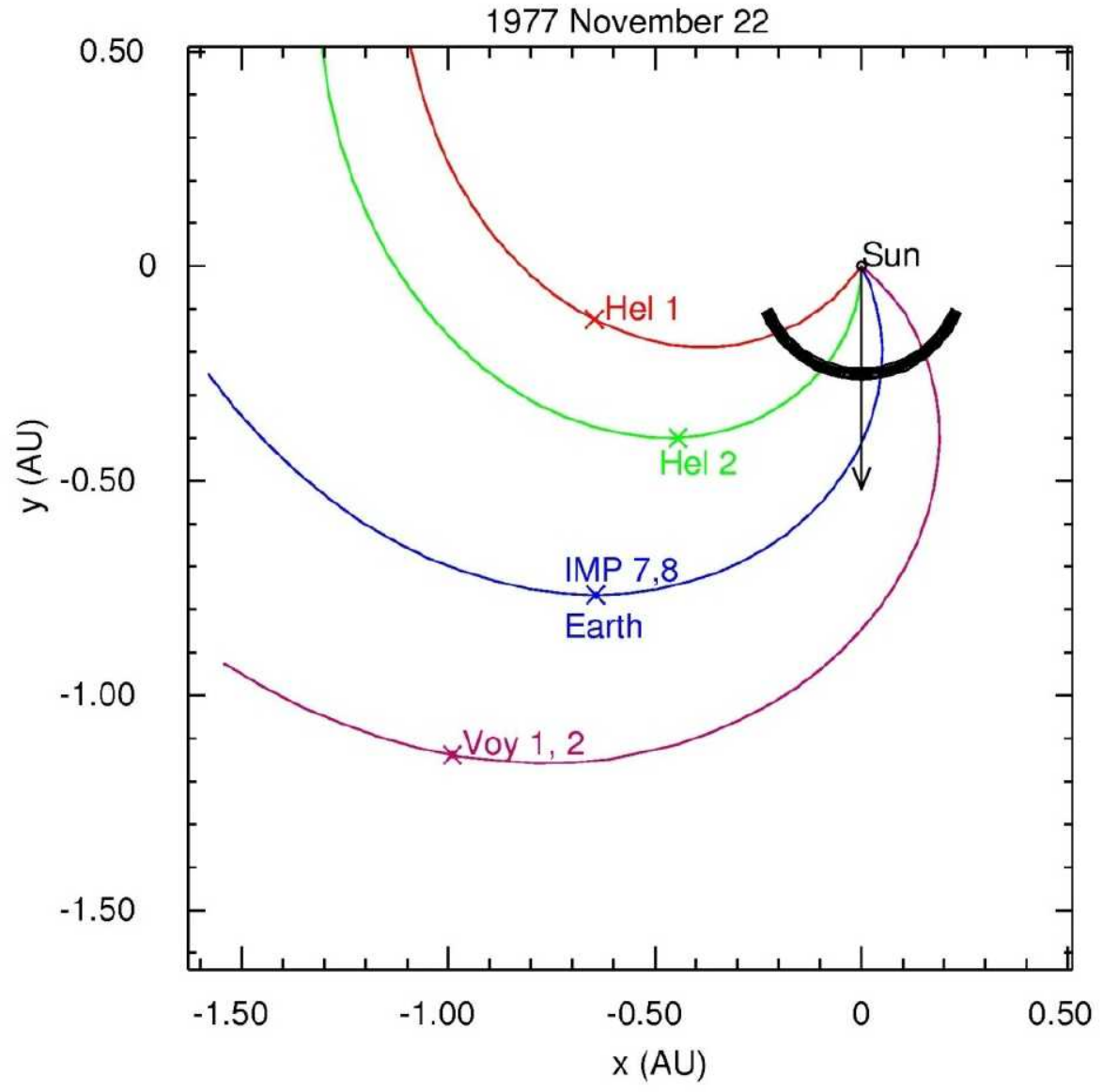

Figure 1 


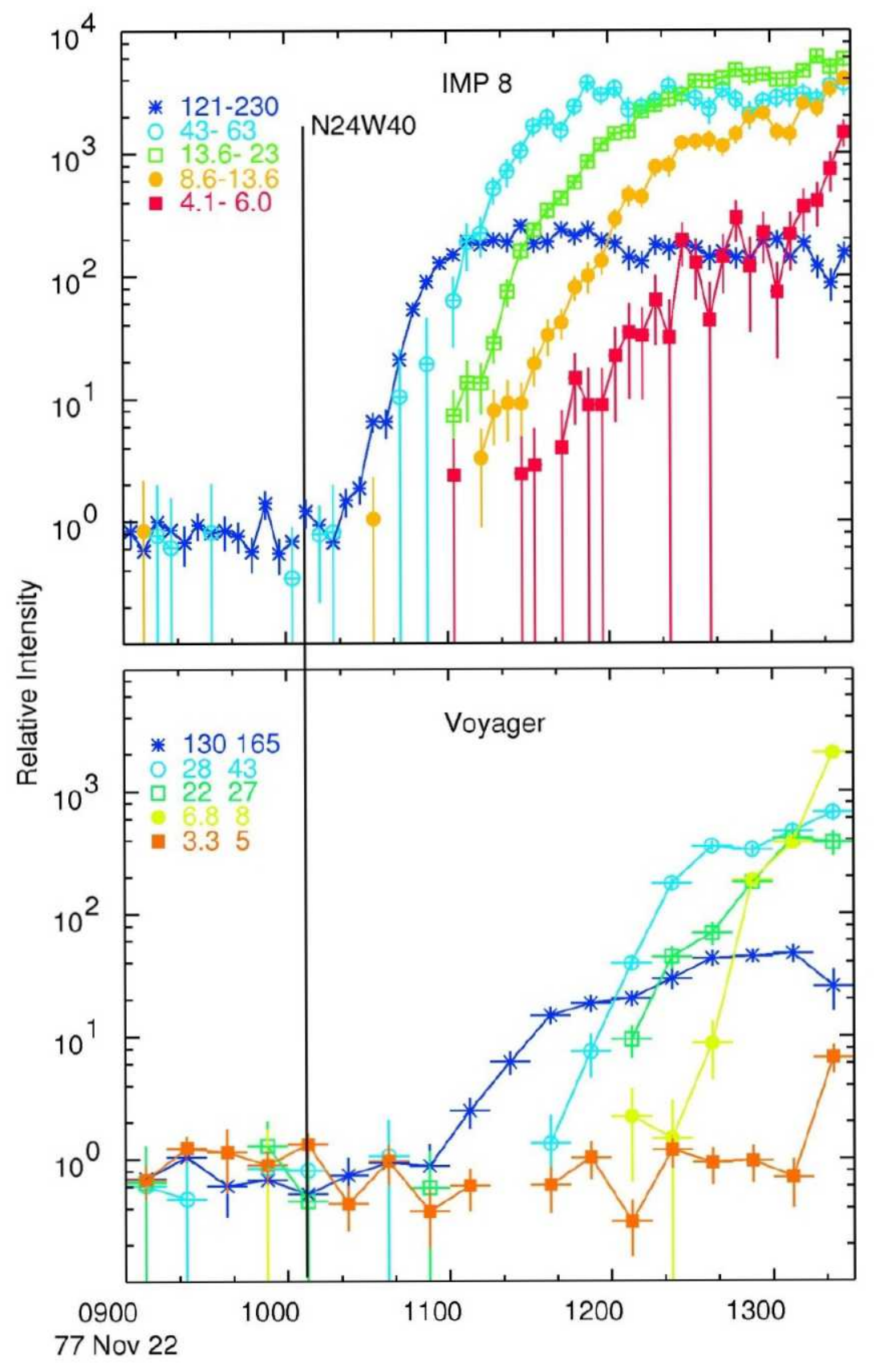

Figure 2 


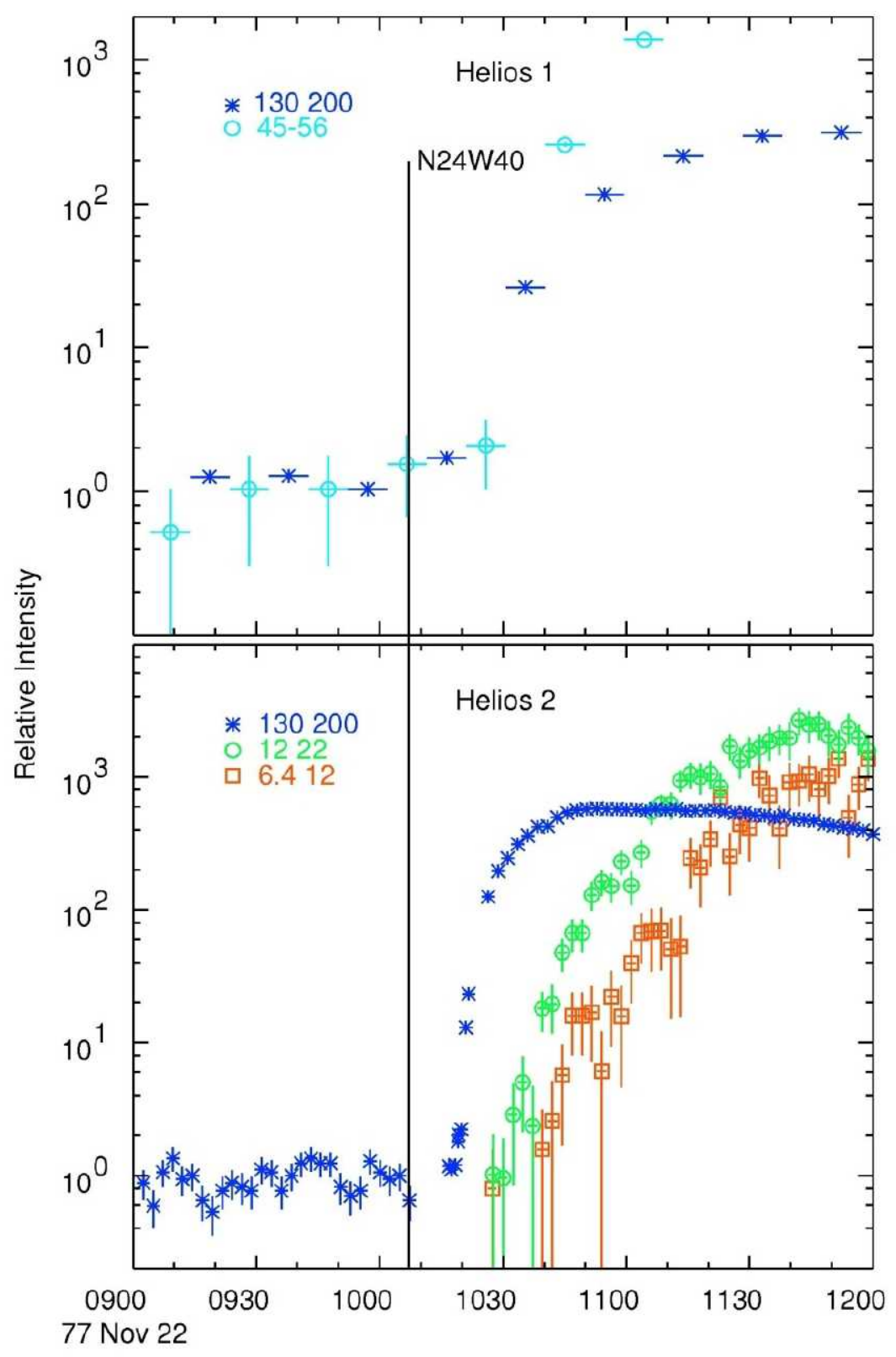

Figure 3 


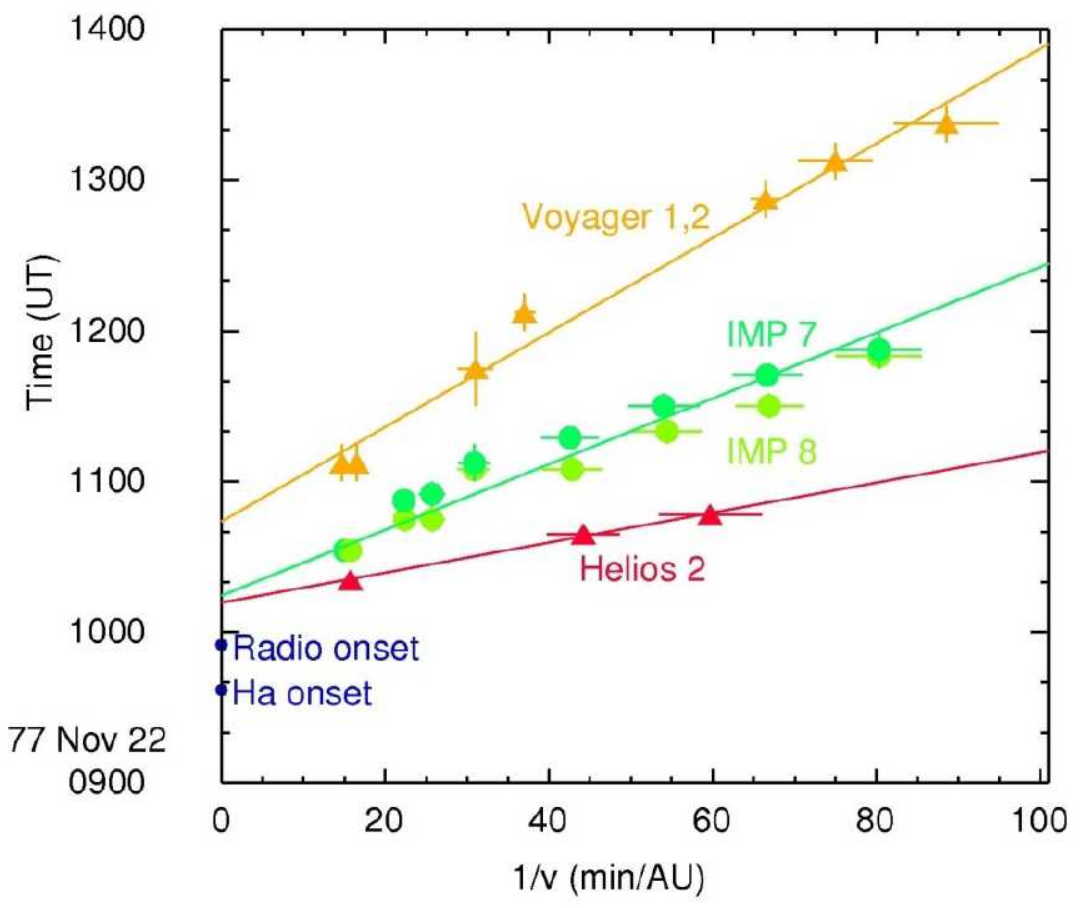

Figure 4. 


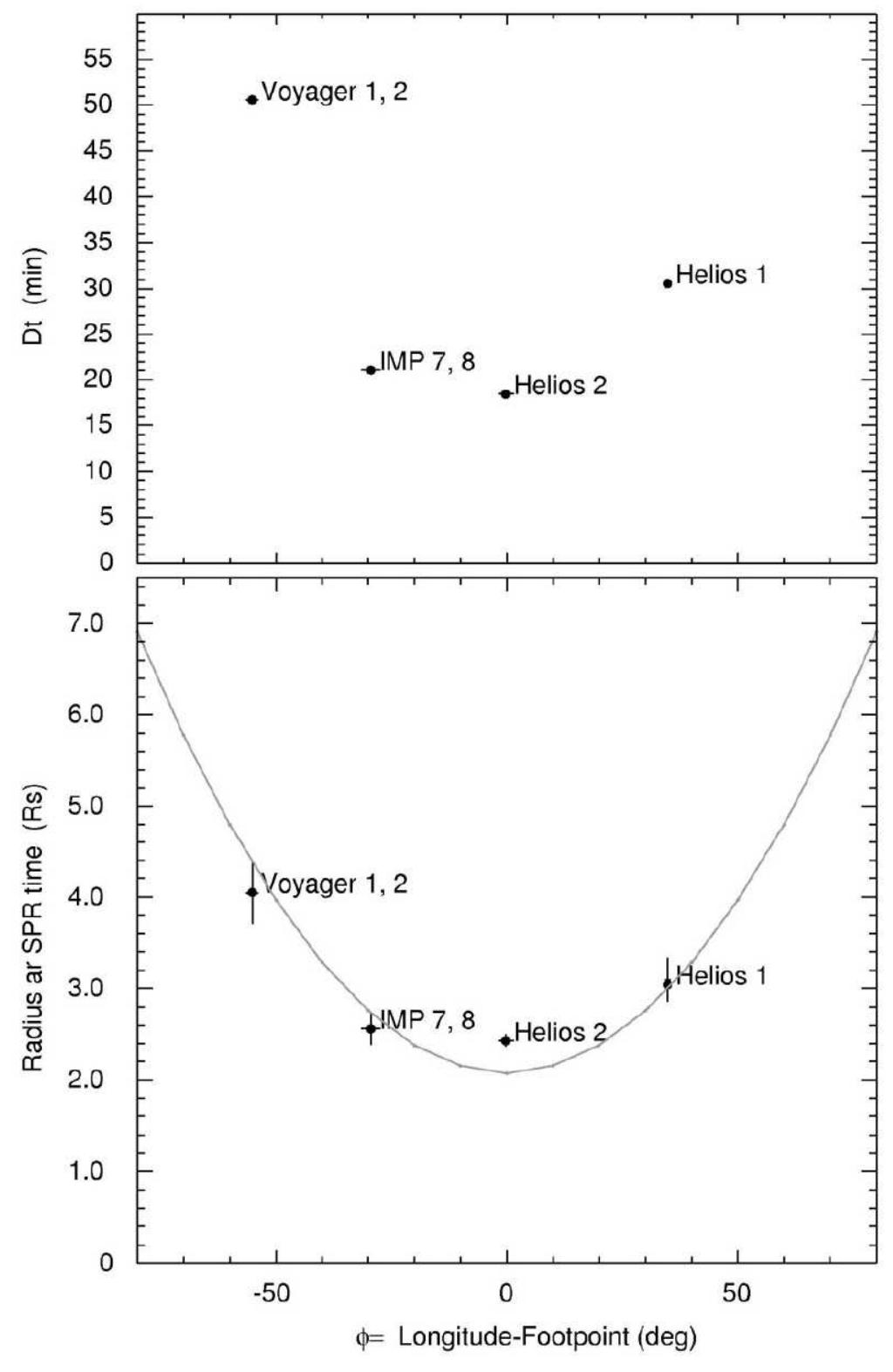

Figure 5 